\title{
Contexto socioeconómico sobre el rendimiento académico del estudiante mediante la investigación como estrategia pedagógica ${ }^{1}$
}

\section{Socio-economic context on the academic performance of the student through research as a pedagogical strategy}

DOI: http://dx.doi.org/10.17981/cultedusoc.9.1.2018.13

Fecha de recepción: 01/05/2018. Fecha de aceptación: 02/08/2018

Marlie Atzene Tique-Gutiérrez ${ }^{2}$ (iD

Juan Andrés Camacho-Anaya; Yenny Del Carmen Segura-Guevara;

Ramiro Orozco-Torregrosa; Lileiva Luz Ortega-Moreno; Mileima Iriarte-Ariza; Jaqueline Martínez-Rudas; Judith Gutiérrez-Pérez; Never Alfonso Cáceres-Fernández ${ }^{3}$

Para citar este artículo

Tique-Gutiérrez, M., Camacho-Anaya, J., Segura-Guevara, Y., Orozco-Torregrosa, R., Ortega-Moreno, L., Iriarte-Ariza, M., Martínez-Rudas, J., Gutiérrez-Pérez, J. y Cáceres-Fernández, N. (2018). Contexto socioeconómico sobre el rendimiento académico del estudiante mediante la investigación como estrategia pedagógica. Cultura. Educación y Sociedad 9(1), 171-180. DOI: http://dx.doi.org/10.17981/cultedusoc.9.1.2018.13

\section{Resumen}

La institución educativa 23 febrero está inmersa en un contexto social donde convergen problemáticas socioeconómicas donde los alumnos les falta tranquilidad y estabilidad en su vida familiar, factores propician un clima de inseguridad afectiva poco acta para una buena adaptación escolar. El estudio busco identificar la influencia del contexto socioeconómico sobre el rendimiento académico del estudiante mediante la Investigación como Estrategia Pedagógica (IEP) en la IED 23 de febrero. Se guio bajo el enfoque cualitativo, con un diseño de Investigación Acción Participación (IAP). Se pudo constatar, que el comportamiento socio cultural de los niños y jóvenes de sectores vulnerables se hace evidente en la cotidianidad escolar puesto que generan comportamientos como: la constante indisciplina, desmotivación, agresiones físicas y verbales, problemas emocionales, deserción, reprobación, la distracción, el poco interés por el aprendizaje, entre otras situaciones, lo cual afecta el proceso educativo que imparten los docentes de distintas instituciones educativas y la atención de los educandos en general.

Palabras clave: contexto socioeconómico, rendimiento académico, investigación como estrategia pedagógica.

\begin{abstract}
The educational institution 23 February is immersed in a social context where socioeconomic problems converge where students lack tranquility and stability in their family life, factors foster a climate of affective insecurity little record for a good school adaptation. The study sought to identify the influence of socioeconomic context on student academic performance through Research as a Pedagogical Strategy (IEP) in FDI February 23. It was guided under the qualitative approach, with an Investigation Action Participation (IAP) design. It was found that the socio-cultural behavior of children and young people from vulnerable sectors is evident in the daily school as they generate behaviors such as: constant indiscipline, demotivation, physical and verbal aggression, emotional problems, desertion, reprobation, distraction, little interest in learning, among other situations, which affects the educational process taught by teachers from different educational institutions and the attention of students in general.
\end{abstract}

Keywords: socioeconomic context, academic performance, research as a pedagogical strategy.

\footnotetext{
${ }^{1}$ Este artículo ha sido derivado del Programa de Fortalecimiento de la Cultura Ciudadana y Democrática CT+I a través de la IEP apoyada en TIC en el Departamento de Magdalena: CICLON

${ }^{2}$ Docente de la Institución Educativa Departamental 23 de Febrero y líder del grupo de investigación Investigadores ventitreistas. Correo de correspondencia: marlieatzene@gmail.com.

${ }^{3}$ Docentes de la institución educativa 23 de febrero, Sede principal y miembros del grupo de investigación Investigadores ventitreistas.
} 


\section{Introducción}

El elemento más importante de la sociedad es la familia, la cual es responsable del comportamiento y del tipo de disciplina a la que están sometidos los jóvenes y niños, muchas de las familias de contextos vulnerables son hogares disfuncionales (padres separados o divorciados), padres que dejan a sus hijos a terceros que los cuiden o viendo programas de televisión sobre violencia ya que no existe un adulto que los oriente. Esto cimenta el comportamiento de los jóvenes frente a sus compañeros escolares y miembros de la familia ya que se ha convertido en una forma de interacción, más que habitual de convivencia diaria.

La clase social, determinada por el ingreso familiar, el tipo y ubicación de la vivienda, la escolaridad, el trabajo que desempeñan los padres, los vínculos que tienen con los hijos, la opinión que los padres tienen de sí mismos y la percepción que tienen acerca su dinámica familiar, son factores importantes en el desenvolvimiento social del alumno, basado en estas apreciaciones el estudio tuvo como objetivo identificar la influencia del contexto socioeconómico sobre el rendimiento académico del estudiante mediante la Investigación como estrategia pedagógica (IEP) en la IED 23 de Febrero del Municipio de Fundación Magdalena.

Uno de los factores que influyen en el comportamiento es el componente sociocultural al que pertenecen los jóvenes de estos sectores donde ciertos comportamientos de comunicación no asertiva se han naturalizado entre compañeros, convirtiéndose es un problema que incide en la convivencia y el desarrollo académico y afectivo. Teniendo en cuenta que los niños y jóvenes de poblaciones vulnerables provienen de distintos contextos sociales los cuales por naturaleza se encuentran expuestas a la exclusión.

Los estudiantes de la institución en su mayoría presentan indisciplina, desmotivación, agresiones físicas y verbales, problemas emocionales, deserción, reprobación entre otras situaciones. Lo anterior afecta el rendimiento académico, por lo que se consideró necesario investigar los contextos donde se desenvuelven para determinar su posible incidencia, con el propósito de sensibilizarlos, pero también verificar si ciertos factores inciden en su desenvolvimiento escolar para así replantear las practicas pedagógicas en las aulas o si es necesario la ayuda profesional para focalizar los casos que requieran de atención especial.

Además, la indiferencia docente y del cuerpo directivo, ante dicha problemática, sabiendo que los alumnos se encuentran inmersos en un contexto altamente vulnerable, donde no han existido propuestas investigativas anteriores en el plantel que hayan buscado soluciones de fondo o minimicen el impacto negativo de este tipo de acciones y conductas, buscando soluciones a través de manuales de convivencia más represivos que al final terminan agudizando los problemas personales de los estudiantes, dejándolo inmerso en los conflictos sociales que le rodean, tampoco se han revisado estrategias pedagógicas, didácticas y motivacionales utilizadas por los docentes si verdaderamente hacen de las aulas un lugar agradable para los estudiantes o por el contrario se convierta en un nuevo problema que se le suma a los que ya trae desde su hogar.

Cabe destacar que estas problemáticas si no se toman los correctivos pertinentes, se agudizaran y el trabajo de formación que hace el docente cada día será más difícil, los índices de deserción 
aumentaran, las agresiones físicas, verbales serán más frecuentes y la calidad educativa se vería afectada seriamente. Un estudio y análisis del contexto que rodea a cada uno de los estudiantes permitiría replantear las estrategias pedagógicas - didácticas de los docentes, una mayor comprensión de los comportamientos y una intervención acertada por parte de la institución en aquellos casos donde se requiera ayuda profesional.

Es de suma importancia e indudable, que el elemento más importante de la sociedad es la familia, tanto que, es responsable del comportamiento y del tipo de disciplina a la que están sometidos los jóvenes y niños, muchas de las familias de contextos vulnerables son hogares disfuncionales (padres separados o divorciados), padres que dejan a sus hijos a terceros que los cuiden o viendo programas de televisión sobre violencia ya que no existe un adulto que los oriente. Esto cimenta el comportamiento de los jóvenes frente a los miembros de la comunidad educativa, convirtiéndose en una forma de interacción habitual.

Teniendo en cuenta que es fundamental la parte social y afectiva de los estudiantes en su rendimiento académico. (Suarez \& Lourdes, 2015) definen diferentes variables que influyen en este de los cuales menciona la parte intelectual, personal, los hábitos de estudio, el auto-concepto y el contenido. Así mismo, atribuye al contexto social una variable fundamental como lo es la familia entendida esta como el núcleo más importante a nivel socio - ambiental ya que aporta al individuo distintos procesos en su construcción individual y colectiva, cada estudiante tiene una herencia genética, y la familia es el primer proceso de socialización que crea la base de la personalidad, aprendiendo los primeros roles, modelos de conducta, imagen sobre sí mismo y normas, acciones las cuales pondrá en práctica en su vida futura.

Dentro de la familia se encuentran diferentes variables, entre estas la estructura y la configuración familiar, el origen y la clase social (nivel socio-económico), el clima educativo familiar (la atención de los padres a los estudios de sus hijos) y el clima afectivo-familiar. Dentro de la variable familia, la que más peso tiene, referente al rendimiento académico, es el clima educativo familiar. Los modelos de familia con el pasar del tiempo han producido cambios (monoparentales, ensamblada, extensas etc.), las dinámicas sociales también han cambiado lo cual ha dado origen a nuevas familias, de acuerdo a esto las conductas y la crianza establece estilos educativos en las familias que determinan el comportamiento de los hijos reflejados en su contexto y en la escuela.

\section{Influencia del contexto socioeconómico sobre el rendimiento académico}

El estudio del contexto socioeconómico en el cual se desenvuelve el alumno ha sido investigado por diversos autores intentando explicar que esos contextos independientes a la escuela contribuyen de forma directa en el desempeño académico, en este sentido se explica que esas variables personales del alumno a las que se hace referencia son el contexto social, familiar, económico y psicológico.

En consideración a esto Gaxiola, González y Contreras, (2012) realizaron una investigación acerca de las condiciones contextuales en las que se desenvuelven los alumnos teniendo como objeto de estudio las metas y las condiciones sociales de los estudiantes para verificar su influencia en el desempeño académico 
como instrumentos de medición utilizaron escalas validadas que les permitieron estudiar las variables, teniendo como resultado que las metas de los estudiantes influyen directamente en su rendimiento académico ya que los estudiantes se fijan objetivos propios en su aprendizaje; en cuanto a el contexto social se encontró que disminuyen el rendimiento académico si se tienen en cuenta aspectos como las amistades de riesgo, que generarían un impacto negativo en la construcción académica del adolescente.

Con relación a esto Miquel, Expósito, y Sempere, (2014), analizaron determinantes sociales sobre el rendimiento académico del estudiante entre sus variables tuvieron en cuenta el nivel socio económico, nivel familiar y la estructura de esta. Para ello utilizaron un cuestionario en el que hicieron preguntas de datos personales y familiares los resultados obtenidos dieron como resultado que los alumnos con mayor rendimiento académico son aquellos que pertenecen a familias amplias y con un nivel económico alto.

En el marco de las observaciones anteriores Erazo, (2012) elaboro un escrito sobre el rendimiento académico tratando este como un fenómeno de múltiples relaciones y complejidades teniendo en cuenta las variables personales del alumno y encontró que estas variables afectan directamente el rendimiento académico y por ende la nota del estudiante.

Teniendo en cuenta que es fundamental la parte social y afectiva de los estudiantes en su rendimiento académico Fernández (2013), define diferentes variables que influyen en este de los cuales menciona la parte intelectual, personal, los hábitos de estudio, el autoconcepto y el contexto. Todo lo anterior, hace referencia al conjunto de características, (físicas, intelectuales, afectiva, sociales, etc.) que construyen la imagen que el sujeto tiene sobre sí mismo, pero que se edifican a lo largo de toda su vida.

Fernández (2013), atribuye al contexto social a una variable fundamental como lo es la familia, entendida esta como el núcleo más importante a nivel socio ambiental ya que aporta al individuo distintos procesos en su construcción individual y colectiva, cada estudiante tiene una herencia genética, y la familia es el primer proceso de socialización que crea la base de la personalidad, aprendiendo los primeros roles, modelos de conducta y la imagen sobre sí mismo, el cual también aprende normas, acciones las cuales pondrá en práctica en su vida futura.

Dentro de la familia se encuentran diferentes variables, entre estas la estructura y la configuración familiar, el origen y la clase social (nivel socio-económico), el clima educativo familiar (la atención de los padres a los estudios de sus hijos) y el clima afectivo-familiar. Dentro de la variable familia, la que más peso tiene, referente al rendimiento académico, es el clima educativo familiar. Fernández (2013), cuando se refiere a contexto social toma en cuenta a la familia como la influencia que tiene en el contexto por el hecho de ser considerada célula social, que protege a los individuos de forma individual y de las relaciones con el otro y es la que ayuda al adolescente a poderse transformar en adulto.

Pero, aun así, aunque la familia protege puede también ser la causa de múltiples problemas ya que es un organismo vivo que nace, crece, se desarrolla y también muere, es el núcleo para la procreación de los hijos y de poder educarlos, esta también tiene otro tipo de funciones que si no se dan correctamente la familia fracasa, por ser el núcleo de la transmisión de valores éticos y morales, de enseñar a 
vivir, enseñar a relacionarse con el resto de la sociedad.

Debido a los cambios que se han producido en la familia, las dinámicas sociales y los modelos de familia han cambiado lo cual ha dado origen a nuevas familias (monoparentales, ensamblada, extensas etc.) de acuerdo a esto las conductas y la crianza establece estilos educativos en las familias que determinan el comportamiento de los hijos reflejados en su contexto y en la escuela. En conclusión, Fernández (2013) determina que es difícil reconocer las variables que influyen en el rendimiento académico; en cuanto a la variable del contexto social, hace énfasis en la familia como el factor más determinante de las conductas y la relación que tienen los individuos con el entorno, pero no enfatiza específicamente que este sea el factor determinante en el rendimiento académico.

Por su parte en cuanto al contexto social hace referencia al entorno físico o simbólico donde el individuo se relaciona con otras personas, este puede abarcar todos los factores culturales, económicos, históricos, etc. que forman parte de su identidad y realidad. El contexto social también abarca el pasado, el presente y el futuro de las personas enmarcado por la influencia de otros. (Mora, Sandoval, \& Acosta, 2013).

Mientras el contexto familiar es el entorno eminentemente existencial donde suelen ocurrir un gran número de experiencias que inducen vivencias íntimas y primordiales derivadas de la convivencia cotidiana con la familia; provee un espacio psicosocial en el que los niños y las niñas obtienen los elementos distintivos de la cultura y las normas sociales que permiten su integración en la sociedad. Y el contexto económico implica las condiciones de producción que comprenden las actividades productivas como los medios, formas y relaciones de producción de ingresos economicos, (Huaynate, \& Anshiri, 2014).

Quienes investigan sostienen que el rendimiento académico hace referencia a la evaluación del conocimiento adquirido en el ámbito escolar. Entendiendo que un estudiante con buen rendimiento es aquél que obtiene calificaciones positivas en las diversas evaluaciones que se realizan durante el periodo escolar de acuerdo a cada asignatura, sin embargo, este no solo se mide a través de las evaluaciones sino de la forma en la que el estudiante se desempeña en las estrategias pedagógicas propuestas por los docentes.

Las estrategias pedagógicas son todas las acciones realizadas por el docente, con el fin de facilitar la formación y el aprendizaje de los estudiantes. Componen los escenarios curriculares de organización de las actividades formativas y de la interacción del proceso enseñanza y aprendizaje donde se logran conocimientos, valores, prácticas, procedimientos y problemas propios del campo de formación, (Bravo, 2008).

Las estrategias pedagógicas suministran invaluables alternativas de formación que se desperdician por desconocimiento y por la falta de planeación pedagógica, lo que genera monotonía que influye negativamente en el aprendizaje. Sobre la base de las consideraciones anteriores una estrategia pedagógica es una guía de acción, en el sentido de que orienta a la obtención de ciertos resultados. La estrategia da sentido y coordinación a todo lo que se hace para llegar a la meta. Desde la experiencia docente las estrategias son sensibles a la modificación constantemente a partir de los propios cambios que se vayan 
operando en el objeto de estudio. Estas responden a los objetivos definidos para los estudiantes en cada nivel de educación, (Mora, et al, 2013).

En la actualidad, existen diversas propuestas metodológicas que incluyen enfoques basados en investigación para la integración de los contenidos curriculares, entendidos como la forma de materializar lo que se hace y como se implementa. Donde surgen las pedagogías basadas en investigación, la cual incluye la propuesta del programa Ondas de Colciencias con la Investigación como Estrategia Pedagógica (IEP), fundamentada como una perspectiva que toma elementos de la pedagogía, el enfoque socio-crítico y una metodología liberadora e integradora. (Mejía y Manjarrés, 2010).

Actualmente la sociedad del conocimiento ha transformado el mundo educativo, donde la ciencia, la tecnología y la innovación inciden en diferentes de forma significativa en la vida de los estudiantes, hecho que ha producido un cambio en los pensamientos y acciones de los individuos frente al proceso de enseñanza. (Cárdenas \& Angulo, 2016)

En ese mismo sentido, La IEP se da una nueva constitución de subjetividad de estos tiempos, en los cuales las culturas infantiles y juveniles, en ese sentido de ser nativos digitales, no solamente hacen su uso, sino que impugnan esa desigualdad en su práctica pedagógica, interpelan la legitimidad de ella y construyen horizontes de posibilidad en donde su práctica grupal le muestra que hay futuro compartido y que él aquí y ahora, con esa colectividad con la que trabaja, lo está construyendo y le permite pensar esos cambios y esas transformaciones porque los comienza a vivir en su vida.

En la misma dinámica, el maestro y la maestra que trabajan con la IEP comprenden las posibilidades de esas transformaciones y se alimentan de ese ejercicio que realizan con sus grupos para producir los cambios y transformaciones en la esfera de su práctica pedagógica, e inician la marcha no por una ley o un mandato, sino porque encuentran allí que pueden ser educadores de otra manera, sin miedo, en búsqueda, sin certezas, pero con la compañía del grupo con el cual van reelaborando su práctica. Ahí, en la esfera del mundo micro -social van surgiendo los gérmenes de las nuevas colectividades que dan forma a la utopía de estos tiempos en educación, participan en ellas sabiendo que es un campo en construcción, y ambos son parte de ello, haciendo real y concreta la idea de "vida buena", planteada por nuestros grupos originarios como una manera de mantener la unidad cultural.

Todo lo anterior, requiere reconocer la importancia de la investigación en la sociedad, a la vez que se develan los intereses presentes. En su práctica forja una mirada crítica, que en coherencia con los tiempos presentes se ve obligada no solo a la denuncia, sino también a apropiarse de ella como una realidad muy importante en esta época para construir propuestas que a la vez que promuevan su apropiación práctica crítica, sirva no solo en modernizar, sino, ante todo, para transformar contextos, culturas, epistemologías, y redirigir sus escenarios de poder construir subjetividades y ciudadanías que reconociendo el lugar de lo local se abran a un tiempo- 
espacio global y a las nuevas mediaciones científico-tecnológicas de este tiempo para hacer concreta la construcción sociedades más democráticas, justas y humanas, así como la necesidad de un planteamiento sobre ella en cualquier proyecto emancipador.

En los departamentos y municipios, los actores optan por desarrollar la propuesta; es decir, constituyen redes (sociales, políticas, de actores institucionales) que hacen real esa búsqueda y se coordinan para concretarla en los diferentes territorios y espacios de sus respectivas localidades. Estas redes han reconocido la necesidad de transformar los procesos de la educación, de la relación entre adultos y niños y niñas, así como de las relaciones sociales que se mediatizan en estas prácticas.

Por ello, esa opción se desarrolla en el contexto, con las particularidades de la cultura, desde las subjetividades constituidas. Todas ellas en una relación: organizaciones sociales, subjetividades, prácticas sociales, dinamizando un escenario de individuación, en donde la integralidad de lo humano (razón, emoción, acción, intereses) debe ponerse en juego para reconocer que nos hacemos humanos en la diferencia y en una interacción conflictiva con los otros y sus realidades.

En el caso de la IEP, se parte de la pregunta del sentido común de los niños, niñas y jóvenes para reelaborarla en la perspectiva de la educación popular, que reconoce la existencia de saberes comunes y elaborados así en la negociación con los conocimientos disciplinares, y de las maneras como se correlacionan en la negociación cultural (Mariño, 2010).

\section{Metodología}

\section{Diseño}

El trabajo de investigación es de enfoque cualitativo, de tipo Investigación Acción Participación (IAP). La investigación cualitativa busca conocer e interpretar la realidad de los participantes a través de sus propias experiencias, entregando una información subjetiva del fenómeno de estudio. (Hernández, Fernández, y Baptista, 2010). En la IAP el conocimiento de la realidad no se descubre ni se posee es el resultado de la trasformación subjetiva y objetiva de el mismo proceso de investigación, en consideración a esta la validez del conocimiento se da por la capacidad que posee para orientar la trasformación de una comunidad o grupo mejorando la calidad de vida de sus integrantes. La IAP es un proceso educativo de autoformación en el cual las personas que pertenecen al grupo sobre quienes esta la realidad estudiada, tienen una participación directa en el proceso de investigación y en la producción de conocimiento de su realidad.

\section{Participantes}

La unidad de análisis estuvo conformada por ochenta (80) estudiantes con edades que oscilaron entre los quince (15) y dieciocho (18) años de edad, de los grados decimo $\left(10^{\circ}\right)$ y once $\left(11^{\circ}\right)$ de bachillerato de la Institución Educativa 23 de febrero del municipio de Fundación Magdalena, los participantes seleccionados viven en estratos socioeconómicos muy bajos, en sectores aledaños a la institución donde se presentan situaciones de violencia, delincuencia juvenil y micro-tráfico de sustancias psicoactivas. Cabe resaltar que los sujetos diligenciaron los requisitos de consentimiento informado. 


\section{Técnicas e instrumentos}

En cuanto a los instrumentos de recolección se utilizó el diario de campo, este es un instrumento que ayuda al investigador a inmiscuirse de forma total en el ambiente con el cual se va a encontrar, es un proceso de hechos, recolección de datos y análisis. El investigador entiende a los participantes y no se dedica únicamente a registrar hechos. El diario de campo es parte de un registro cronológico que se desarrolla mediante la sesión compartida con los infantes, es donde se describe detalladamente los aspectos positivos como negativos colocando significado a cada uno de ellos como un recurso de mejoras en todas las áreas con el grupo a trabajar considerando los recursos manejados y utilizados. Un cuaderno donde se va a descubrir la manera de expresar y escribir frente a la relación que se tiene con los infantes, es un momento de no cohibir lo vivido y las experiencias obtenidas.

\section{Procedimiento}

La metodología del proyecto de investigación se realizó a partir de los recorridos de las trayectorias de indagación, basados en la integración de la IEP apoyada en TIC al aula, articulado al plan de estudios, desde el área de sociales se estructuraron temáticas relacionadas con el contexto socioeconómico, estudiando las dinámicas familiares y sociales de los estudiantes de la institución, la finalidad de este proceso investigativo, es generar una trasformación social y educativa a través del aprendizaje en contexto, involucrando intereses, motivaciones y realidades de los estudiantes para generar conocimiento científico, donde se establecieron cinco (5) momentos que especifican en la ejecución y las metas trazadas para darle solución a la pregunta problema planteada. A continuación, se presentan los diferentes momentos:

- Momento (1): selección y análisis de la población sujeto de estudio.

- Momento (2): integración de la temática mediante la IEP al aula de clases, se integraron grupos estudiantiles para seleccionar problemáticas relacionadas el contexto socioeconómico. Los estudiantes se plantearon preguntas y con el acompañamiento del docente realizaron el proceso de indagación mediante herramientas virtuales y entrevistas a la comunidad.

- Momento (3): socialización de los resultados de las indagaciones de estudiantes en el aula de clase.

- Momento (4): análisis de los resultados para observar la influencia de variables contextuales en el rendimiento académico de los estudiantes.

- Momento (5): propagación y divulgación de los resultados.

\section{Resultados}

Los resultados de la investigación evidenciaron que existe una influencia bidireccional en entre el contexto socioeconómico de los estudiantes y su rendimiento académico, esto fue posible mediante la integración de la IEP al aula de clase, donde estos realizaron el proceso de indagación guiado por los docentes y diseñaron entrevistas semiestructuradas para la recopilación de datos, estas las aplicaron a sus compañeros y familias, para describir el contexto de la población.

$\mathrm{Al}$ comparar los resultados de las entrevistas realizadas por los estudiantes 
con los boletines del segundo período se pudo comprobar la incidencia del factor familiar y económico en su rendimiento académico. Por ejemplo, aquellos estudiantes que afirman tener una relación regular o mala con la familia tienen bajo rendimiento académico; también se pudo comprobar que con relación a los ingresos mensuales por familia y los servicios con los que puede contar el estudiante en enriquecer su labor académica, aquellos estudiantes que afirmaron tener menos de estos recursos poseen un desempeño académico más bajo en comparación los que poseen ingresos familiares superiores y herramientas para trabajar.

Finalmente, al hacer referencia al contexto con las familias de diversas dinámicas sociales, como las descritas en esta investigación, carentes de oportunidades económicas, afianzan aún más, las desigualdades entre los estudiantes que cuentan con los mismos derechos e igualdades de todos, el derecho fundamental a una educación equitativa y de calidad, que no logran obtener acceso a esta por las mismas dinámicas referidas con anterioridad. La realidad que tienen cientos de familias en condiciones desfavorables existentes en nuestro país.

Una vez obtenido los resultados parciales del proyecto, se aprovechó en concretar una reunión general de docentes con el propósito de socializarles el recorrido del proyecto desde inicio hasta la aplicación de los instrumentos finales (encuesta, entrevista), mostrarles la realidad que circunda a los estudiantes con evidencias fotográficas obtenidas de las visitas domiciliarias , la socialización de las gráficas producto de las encuestas y compararlos con los informes académicos con el propósito de determinar si se alcanzó o no el propósito de la iniciativa.

\section{Referencias bibliográficas}

Bravo, H. (2008). Estrategias pedagógicas. Córdoba. Universidad del Sinú.

Erazo, A. (2012) Rendimiento académico un fenómeno de múltiples relaciones y complejidades; Revista Vanguardia Psicológica; Universidad Manuela Beltrán, Vol. 2.

Fernández, C. (2013). Rendimiento escolar y contexto social en educación primaria. (Trabajo de pregrado)

Gaxiola, J., \& Contreras, Z. (2012). Influencia de la residencia metas $y$ contexto social en el rendimiento académico de bachilleres. Revista Electrónica de Investigación Educativa. Vol. 14.

Hernández, R., Fernández, C., \& Baptista, P. (2010). Metodología de la investigación. México. Editorial Mc Graw Hill.

Huaynate, V., \& Anshiri, J. (2014). Factores familiares y sociales que determinan el internamiento por actos delictivos de los adolescentes albergados en el centro de atención residencial hogar para niños y adolescentes san josé inabif derivados por los juzgados de familia de la corte superior de justicia de la libertad, durante el año 2013.

Mejía, M., y Manjarrés M. (2010). Las pedagogías fundadas en la investigación. Búsquedas en la reconfiguración de la educación. Revista Internacional Magisterio 42: 16-26. Colombia.

Miquel, J., Expósito, M., \& Sempere, S. (2014). Determinantes del rendimiento académico en los estudiantes de grado. Un estudio en administración y dirección de empresas. Revista de investigación educativa, 32(2), 379392. 
Mora, M., Sandoval, Y., \& Acosta, M. (2013). Estrategias pedagógicas y didácticas para el desarrollo de las inteligencias múltiples y el aprendizaje autónomo. Revista de Investigaciones UNAD, 12(1), 101-128.

Ortega, P. P. (2009). Sujetos y prácticas de la pedagogía crítica. Bogotá: El Búho.
Suarez, C., \& Lourdes, M. (2015). Importancia de la autoestima en niños y niñas de Educación Inicial de 3-4 años en los meses de agosto-noviembre del año lectivo 2015-2016 de la Escuela 18 de octubre.

Torres, A. (2007). La educación popular. Trayectoria y actualidad. Bogotá: El Búho. 